\title{
Evaluating Cases in Legal Disputes as Rival Theories
}

\author{
Pontus Stenetorp ${ }^{1}$ and Jason Jingshi $\mathrm{Li}^{2,3}$ \\ ${ }^{1}$ Graduate School of Information Science and Technology, \\ The University of Tokyo, Tokyo, Japan \\ pontus@is.s.u-tokyo.ac.jp \\ ${ }^{2}$ College of Engineering and Computer Science, \\ The Australian National University, Canberra, Australia \\ ${ }^{3}$ Canberra Research Laboratory, \\ NICTA, Canberra, Australia \\ jason.li@anu.edu.au
}

\begin{abstract}
In this paper we propose to draw a link from the quantitative notion of coherence, previously used to evaluate rival scientific theories, to legal reasoning. We evaluate the stories of the plaintiff and the defendant in a legal case as rival theories by measuring how well they cohere when accounting for the evidence. We show that this gives rise to a formalized comparison between rival cases that account for the same set of evidence, and provide a possible explanation as to why judgements may favour one side over the other. We illustrate our approach by applying it to a known legal dispute from the literature.
\end{abstract}

Keywords: legal argument, legal justification, theory construction, coherence.

\section{Introduction}

In legal disputes each side present their case before the court, outlining the issues, positions, and arguments taken with respect to the issues. The "story" is supported by evidence, which is sometimes explicitly sought by the judge as burden of proof. Each side must explain how the evidence fits their story, though there may be elements of their story that for some reason cannot be verified by evidence or empirical testing.

Similarly in the philosophy of science, rival, possibly incompatible scientific theories must also account for all observations, but empirical testing cannot always be used to differentiate or rank theories, as they make the same empirical claims. One possible measure to evaluate theories is how coherent a theory is in accounting for a given set of observations.

We propose to draw parallels from this notion of coherence to legal reasoning, where we view the cases of the plaintiff and the defendant in a legal dispute as rival theories, and evaluate the cases by measuring how coherent the stories are in their account for the evidence. Intuitively, just as a good scientific theory uses

K. Nakakoji, Y. Murakami, and E. McCready (Eds.): JSAI-isAI, LNAI 6284, pp. 59-72, 2010.
(C) Springer-Verlag Berlin Heidelberg 2010 
only a few credible postulates to explain a large body of evidence, a good "story" in a legal case must account for the evidence using only a few minor assumptions. This provide an alternative view on how the cases can be evaluated and decided, and provides a possible explanation as to why judgements may favour one side over the other, in a formal and structured manner.

The notion of coherence in regard to legal justification is well explored by Hage 3] and Amaya 1]. Equally, there is abundant literature on abductive reasoning with respect to the evidence and the burden of proof by Prakken et al. [10] and Satoh et al. [11. However, the existing literature on coherence is mostly concerned with how a decision can cohere with current law and cases, whereas we are interested in the overall picture of how the "story" of the plaintiff/defendant coheres with the evidence.

In this paper we introduce the notion of coherence in the form proposed by Kwok et al. [5, 6] for evaluating scientific theories. We then propose a possible scheme based on the previous work by Kwok et al. [5, 6, for evaluating the coherence of cases in a legal dispute. This is followed by an example of applying our theory to an actual legal dispute previously formalized by Prakken [8]. In conclusion, we discuss what is implied by our coherence measure and possible future directions of this work.

\section{Coherence of Theories}

Traditionally in the philosophy of science, coherence has always been a criterion in evaluating the quality of scientific theories. The extent of coherence of a theory depends on informal, qualitative notions such as "brevity", "predictive scope" and "tightness of coupling" of the components of the theory.

Kwok et al. [5] proposed a quantitative measure of coherence based on the average utilization of formulas in accounting for observations. Their later work, Kwok et al. [6], better mirrored scientific practice by introducing input and output sets. The proposed measure facilitates the testing of theories with experiments that have varying inputs and outputs, where each theory is expressed as a set of clauses.

When performing a scientific experiment we provide a certain input $I$ and observe a certain output $O$. It is then the objective of a theory $T$ to explain how the input leads to the output. For example, in an experiment verifying the theory of gravity, the input being an object dropped in vacuum, the output would be the measured velocity of the object some time after the drop and the theory provides a link between the input and output. In this section we summarize the approach developed by Kwok et al. 6].

Definition 1 (Support Sets). Given an input set I, an output set $O$, a subset of the theory $T$ being $\Gamma$. Then, $\Gamma$ is a I-relative support set of $O$ if:

1. $\Gamma \wedge I \models O$ and

2. $\Gamma$ is minimal (wrt set inclusion). 
Support sets are the building blocks of the coherence measure. They are the formulas that account for a particular observation for a given input. We denote $S(T, I, O)$ to be the family of all $I$-relative support sets for $O$.

Definition 2 (Utility of a formula). Given an input set $I$, an output set $O$ and a theory $T$. For a formula $\alpha \in T$, the utility of $\alpha$ with respect to T, I, $O$ is given by:

$$
U(\alpha, T, I, O)=\frac{\mid\{\Gamma: \alpha \in \Gamma \text { and } \Gamma \in S(T, I, O)\} \mid}{|S(T, I, O)|} \text { if } S(T, I, O) \neq \emptyset
$$

The support sets give rise to the definition of the utility of a formula of the theory. Informally, this is the relative frequency of occurrence of formula $\alpha$ in the support sets of $T, I, O$. This reflects the contribution of $\alpha$ in $T$ to account for the pair $(I, O)$.

Definition 3 (Coherence of a Theory). The coherence of a theory $T$ with formulas $\left\{\alpha_{1}, \ldots, \alpha_{n}\right\}$ with respect to input observations $I=\left\{I_{1}, \ldots, I_{m}\right\}$ and output observations $O=\left\{O_{1}, \ldots, O_{m}\right\}$ is:

$$
C(T, I, O)=\frac{1}{m n} \sum_{i=1}^{n} \sum_{j=1}^{m} U\left(\alpha_{i}, T, I_{j}, O_{j}\right)
$$

The coherence of a theory is the average utility of the theory's formulas in accounting for all the observations from possibly multiple experiments. This measure has been shown by Kwok et. al. [6] to demolish Craig's trick as shown by Craig [2, where empirical observations are simply added to the theory as exceptions. They showed that such handling of exceptions results in the formulation of highly incoherent theories, since the measure favours theories in which a small subset of the theory accounts for a large body of evidence.

\section{Evaluating Legal Cases}

In this section, we draw a link from scientific disputes between rival theories to ordinary legal disputes between the cases of the plaintiff and the defendant. We treat the "stories" given from both sides as rival theories, each of which can be tested against the evidence presented to the court. Just as scientific theories can be tested over multiple experiments, a case in a legal dispute can be tested by multiple pieces of evidence and testimonies.

In the following sections we will show how the analogy can be made. Our approach enables us to evaluate the coherence of a case by measuring how well the components of the case are utilized when accounting for the evidence and testimonies presented to the court. We assume that all the components of the sets mentioned in the following subsections are in clausal form. 


\subsection{Inputs}

The presentation of evidence and testimonies can be viewed as experiments testing the theory. Hence the input of the experiments are the relevant laws and the mutually accepted state of affairs that are necessary for the theory to entail the output. Laws themselves can not be disputed although their validity for a certain case may very well be questioned.

A mutually accepted state of affairs is a state of affairs that is presented by some participant of the trial, but is not contested by any other participant. This notion is the same as the view on common knowledge given in Walton and Macagno [12. It can thus be deemed that a mutually accepted state of affairs can be regarded as a fact from which one may draw conclusions or aid arguments, even though the truth of such a state of affairs is never proven explicitly to the court. We relax the requirement on inputs to include laws and facts that are not used in deriving the output, as they do not affect the utility of any component of the theory, and hence have no effect on measuring coherence.

\subsection{Outputs}

The outputs are the evidence and testimonies presented to the court. They pose the main problem for any theory since it must explain how an output can be explained using the theory itself and the inputs mentioned in the previous section.

Both the plaintiff and the defendant must account for the observations in the output such as why certain DNA is present at the crime scene, why the witness $x$ testify that $y$ loaned equipment to $z$. Without explaining such circumstances a case may not be considered to fulfil the requirements of the court.

\subsection{Theory}

The theory in the case of a legal dispute is the "story" that is told by one of the sides. It may contain several components to explain the evidence presented to the court, and why the desired outcome holds for the plaintiff or the defendant. The requirement to hold the sought outcome becomes obvious: if one considers the "story", it must in some way justify the conclusion the side hopes for or nothing can be gained from the trial.

The two theories, while arguing for different outcomes, will have to take into account the same laws and mutually accepted state of affairs, which in our framework corresponds to the inputs. But they must also account for the same evidence and testimonies put forth to the court, in our framework the outputs. Making the assumption that they both account for all the facts they are clearly rival theories that account for the same set of evidence but must somehow be differentiated regarding how well they do so.

\subsection{Support Sets and Coherence}

For our measure of coherence we need to observe the $I$-relative support set for the outputs $O$ that is a subset of the theory that accounts for a particular piece 
of evidence $O_{i}$ given input $I_{i}$. The relative frequency of a component of the theory appearing in the support sets give rise to the utility of the component, where coherence is measured as the average utility of the components over all the given evidence.

Intuitively, the support set for a given piece of evidence is how the plaintiff/defendant explain that piece of evidence with respect to the mutually accepted state of affairs and relevant laws presented before the court. The coherence of the theory measures how well the overall "story" of the plaintiff and the defendant explains all the evidence. Any theory that assumes freely without proper support will thus be punished with lower coherence than a theory which can utilize a small amount of assumptions in combination with the inputs to account for a larger body of evidence.

\section{Example}

As an example of how our notion of coherence can be applied in order to evaluate rival legal cases between a plaintiff and a defendant, we apply it to a known legal dispute first formalized by Prakken [8].

Unlike Prakken 8 we simplify the case into sets of clauses to form the basis of the judgement rather than utilizing an argumentation framework to focus on the process of the trial. We will attempt to stay consistent with as much of the previous formalization as possible, differing only when our notion of coherence and our focus on the judgement rather than the process forces us to do so.

\subsection{The Dispute}

The legal case formalized by Prakken [8] is a Dutch civil case from 1978, concerning the ownership of a moveable good, a large tent. The owner of the tent, Mr. van der Velde, put the tent up for sale at the price of 850 Gulden (approx. 380 Euro). Mr. Nieborg, who was a friend of Mr. van der Velde, said that he was interested in buying the tent but could not afford it. Mr. van der Velde made the tent available to Mr. Nieborg, who in return helped Mr. van der Velde to paint his house. Also, Mrs. Nieborg helped Mrs. van der Velde with her domestic work for some time.

Later, Mr. Nieborg claimed that he and his wife had performed enough work for Mr. and Mrs. van der Velde to cover the cost of the tent, thus implicitly claiming that he had now become the legitimate owner of the tent. This angered Mr. van der Velde since he perceived the work performed by Mr. and Mrs. Nieborg as an expression of gratitude for allowing them to use the tent as a loan. He immediately demanded that Mr. Nieborg would return the tent. When his demands were not met, Mr. van der Velde, with assistance, threw Mr. Nieborg's son, who was the person currently occupying the tent, out of the tent and took possession of it.

Some time later, Mr. van der Velde sold the tent to a Mr. van der Weg. Mr. van der Weg paid for the tent by performing work, which was similar to the work 
performed earlier by Mr. and Mrs. Nieborg, for Mr. van der Velde. Mr. Nieborg took his case against Mr. van der Weg to court within a period of time which was less than three years after the repossession of the tent carried out by Mr. van der Velde, a fact that should be noted due to implications in regard to Dutch law.

We present the cases of both Mr. Nieborg and Mr. van der Weg in clausal form. In order to make our clauses as brief as possible, we abbreviate the names of the people as presented in Table 1. We also abbreviate the relevant points in time as presented in Table 2, Both sets of abbreviations conform to those used by Prakken [8. To make the feel of the running text more natural, we will still make use of the full names and points in time.

Table 1. Abbreviations for the participants of the trial

\begin{tabular}{lcl}
\hline Surname & Abbreviation Role \\
\hline Mr. Nieborg & $\mathrm{N}$ & Plaintiff \\
Mr. van der Weg & vdW & Defendant \\
Mr. van der Velde & vdV & Witness \\
Mr. Sluis & $\mathrm{S}$ & Witness \\
Mr. Galtema & $\mathrm{G}$ & Witness \\
\hline
\end{tabular}

Table 2. Abbreviations for the points in time relevant to the trial

\begin{tabular}{cc}
\hline Point in time & Event \\
\hline$t_{1}$ & $\mathrm{~N}$ held the tent \\
$t_{2}$ & Violent events between vdV and $\mathrm{N}$ \\
$t_{3}$ & Time of the trial \\
\hline
\end{tabular}

\subsection{Inputs - Relevant Laws, Mutually Accepted State of Affairs and Consequences}

We will in this section present the relevant laws, mutually accepted state of affairs and consequences, all of which will be presented in clausal form as well as informally in the running text. These clauses will serve as input to be utilized by the respective theories in order to derive the evidence and testimonies which we will refer to as output. This derivation will be done using the theory itself and a subset of the input clauses. The clauses are partially those found in Prakken 8, but with some additions.

One difference to Prakken [8] is that we only consider the law, mutually accepted state of affairs and consequences that are relevant to the final juridical judgement. This difference is due to our focus on the final judgement rather than the process of the trial itself as is the case with an argument framework, thus we can disregard of a more general law that is later refuted in favour of one which for our case applies more specifically. We will point out these special cases when presenting the clauses. 
All laws formalized here were in effect at the time of the trial. Also, just as in Prakken [8] we do not motivate the notion of persistence of ownership that Mr. Nieborg implicitly uses to justify that he indeed is still the owner of the tent at the time of the trial, since according to him no change in ownership has taken place since he took possession of the tent from Mr. van der Velde.

$$
\begin{gathered}
\operatorname{Hold}\left(N, T e n t, t_{2}\right) \\
\operatorname{Hold}\left(v d W, T e n t, t_{3}\right)
\end{gathered}
$$

The first two clauses, 1 and 2 concerns the holder of the tent at different points in time. Clause 1, that Mr. Nieborg held the tent at the time it was taken from him. Clause 2, that Mr. van der Weg now holds the tent. Both parties agrees upon these state of affairs.

$$
\begin{gathered}
\operatorname{Loan}(x, y) \rightarrow \text { TestimonyLoan }(z, x, y) \wedge(x \neq z) \\
\text { FalseTestimonyLoan }(x) \rightarrow \text { TestimonyLoan }(x, y, z) \wedge(x \neq y) \\
\text { Violence }(x, y) \rightarrow \text { TestimonyViolence }(z, x, y) \wedge(y \neq z) \\
\text { FalseTestimonyViolence }(x) \rightarrow \text { TestimonyViolence }(x, y, z) \wedge(x \neq z)
\end{gathered}
$$

The next set of clauses, clause 3 to clause 6. lays forth the logic concerning testimonies, it should be noted that we assume a primitive notion of lying to simplify our set of clauses. It should also be noted that these clauses have no temporal components, as we previously saw for clauses 1 and 2, the reasoning behind this is that the testimonies and events in our particular case do not need any temporal components due to them occurring only once. These four clauses can not be refuted logically and have to be accepted by all parties.

Clause 3 and 5 simply state that if a person $x$ borrowed an item $y$ or violence was inflicted by a person $x$ towards person $y$, then a third person $z$ can deliver a testimony of the event. Clause 4 and 6 provide an alternate mode for explaining each testimony. If a witness is lying, then he would deliver the same testimony that a witness who had observed the events would have delivered.

$$
\operatorname{Hold}(x, y, t) \wedge \neg \operatorname{Loan}(x, y) \rightarrow \operatorname{Possess}(x, y, t)
$$

Clause 7 is a formalization of Dutch law 590 BW. The loan condition is a simplification of the actual text that states that the holder may not be holding it for another person, this change is made to make the clause simpler since holding the item can be derived from a loan in our specific case. The loan condition is an exception added to the more general version of the law that lacks this condition, but since for our case the loan condition is relevant, we observe the more specific law. This constraint makes it possible to disregard the notion of law precedence in Prakken [8] and is justified by us only observing the judgement.

$$
\operatorname{Possess}(x, y, t) \rightarrow \operatorname{GoodFaith}(x, y, t)
$$




$$
\begin{aligned}
\operatorname{Possess}(x, y, t) & \wedge \operatorname{GoodFaith}(x, y, t) \wedge \operatorname{Owner}\left(z, y, t^{\prime}\right) \wedge(x \neq z) \\
& \wedge \text { InvoluntaryLoss }\left(z, y, t^{\prime}\right) \wedge\left(t^{\prime}-t\right)<3 \text { years } \\
& \rightarrow \neg \text { Owner }(x, y, t)
\end{aligned}
$$

Clause 8 is a formalization of Dutch law 589 BW which states that a possessor is presumed to be a possessor of good faith. Clause 9 is a formalization of Dutch law 2014 BW regarding the possession of a good. It covers the special case that $x$ can not be the owner of $y$ if it has occurred an involuntary loss of $y$ from a previous owner $z$ at a time $t^{\prime}$. It also contains the restriction that a maximum of three years must have passed since the time of the involuntary loss $t^{\prime}$ and the current point in time $t$.

\subsection{Evidence Presented}

This section covers all irrefutable evidence presented to the court, these are facts that must be accounted for by any theory in order for a case to be considered valid. This can be done by utilizing a subset of a theory, by calling upon law and/or mutually accepted state of affairs, as presented in section 4.2 . The set of all evidence clauses is referred to as the output set $O$.

$$
\begin{gathered}
O_{1}=\text { TestimonyLoan }(v d V, N, \text { Tent }) \\
O_{2}=\text { TestimonyLoan }(G, N, \text { Tent }) \\
O_{3}=\text { TestimonyLoan }(S, N, T e n t) \\
O_{4}=\text { TestimonyViolence }(v d V, v d V, N) \\
O_{5}=\text { TestimonyViolence }(G, v d V, N) \\
O_{6}=\text { TestimonyViolence }(S, v d V, N)
\end{gathered}
$$

Clauses 10 to 15 are all testimonies delivered to the court. That the testimonies took place is irrefutable, but the fact of them taking place has to be explained by each theory presented in the next two sections 4.4 and 4.5 .

To simplify our clauses we have taken the liberty of stating that the testimonies of violence implied violence towards Mr. Nieborg. In reality the involved party was Mr. Nieborg's son. We have also done the same regarding the testimony of the tent being a loan, what was presented in reality was that Mr. Nieborg expressed gratitude towards Mr. van der Velde for being able to hold the tent for a limited time. This was observed by the witnesses, who gave testimonies to that effect. As described by Prakken [8] the violence towards Mr. Nieborg's son counts as violence towards Mr. Nieborg when legally proving that the loss was involuntary and the expression of gratitude observed by the three witnesses counts as the possession of the tent being perceived as a loan. We have once again simply left out these conclusions and replaced them with the results relevant to the judgement. 


\subsection{The Plaintiff's Case}

To make a case, each party $x$ must construct an input set $I_{x}$ which consists of input subsets $I_{x i}$ corresponding with the observation $O_{i}$ presented in the previous section. The clauses of each subset $I_{x i}$ are clauses from section 4.2 , being formalizations of mutually accepted state of affairs and law.

Each party $x$ also needs to produce a theory $T_{x}$ representing his "story", that together with the input subset $I_{x i}$ will explain the corresponding observation $O_{i}$. A theory may consist of any clauses, as long as it satisfies the previously mentioned condition to satisfy each $O_{i}$ by using $I_{x i}$ as input.

$$
\begin{gathered}
T_{P 1}=\text { Violence }\left(v d V, N, t_{2}\right) \\
T_{P 2}=\text { FalseTestimonyLoan }(v d V) \\
T_{P 3}=\text { FalseTestimonyLoan }(G) \\
T_{P 4}=\text { FalseTestimonyLoan }(S) \\
T_{P 5}=\neg \text { Loan }(N, \text { Tent })
\end{gathered}
$$

The first clause of the plaintiff's theory $T_{P}$, clause 16, is an acceptance of the violent events when the possession of the tent was revoked by Mr. van der Velde since this plays in his favour. However, he is forced to add clauses 17 to 19 since he is unwilling to accept that his work was an expression of gratitude, which was how it was perceived by the witnesses. Not calling the testimonies false would render it impossible for him to claim previous possession, using law $590 \mathrm{BW}$ with its special case (clause 7) and law $589 \mathrm{BW}$ (clause 8). Thus being able to revoke the current hold of the tent by van der Weg using law 2014 BW (clause 9) with its exception which is his own goal and his theory must thus account for this. Clause 20 is included since it is a requirement for him to be able to use law 2014 BW (clause 9) with the special case applied.

$$
\begin{gathered}
I_{P 1}=\{\text { FalseTestimonyLoan }(x) \rightarrow \text { TestimonyLoan }(x, y, z) \wedge(x \neq y)\} \\
I_{P 2}=\{\text { FalseTestimonyLoan }(x) \rightarrow \text { TestimonyLoan }(x, y, z) \wedge(x \neq y)\} \\
I_{P 3}=\{\text { FalseTestimonyLoan }(x) \rightarrow \text { TestimonyLoan }(x, y, z) \wedge(x \neq y)\} \\
I_{P 4}=\{\text { Violence }(x, y) \rightarrow \text { TestimonyViolence }(z, x, y) \wedge(y \neq z)\} \\
I_{P 5}=\{\text { Violence }(x, y) \rightarrow \text { TestimonyViolence }(z, x, y) \wedge(y \neq z)\} \\
I_{P 6}=\{\text { Violence }(x, y) \rightarrow \text { TestimonyViolence }(z, x, y) \wedge(y \neq z)\}
\end{gathered}
$$

Since it is an important point, we will once again stress that the input set $I_{x}$, unlike the theory set $T_{x}$, has the restriction that it can only consist of laws and mutually accepted state of affairs. This has significant consequences for our notion of coherence, which we will observe in the coming sections.

For his input set $I_{P}$ the plaintiff alternates between clause 4 that implies that a testimony is a lie and clause 5 that implies that a testimony is accurate. This in combination with his theory $T_{P}$ is enough to prove each $O_{i}$ using the corresponding $I_{P i}$, thus completing his task. 


\subsection{The Defendant's Case}

The defendant's case is very similar to that of the plaintiff which we presented in the previous section. But the minute differences will have effects on how it interacts with our notion of coherence.

$$
\begin{gathered}
T_{D 1}=\operatorname{Violence}\left(v d V, N, t_{2}\right) \\
T_{D 2}=\operatorname{Loan}(N, \text { Tent })
\end{gathered}
$$

In his theory $T_{D}$, the defendant has no need to discredit the violent events taking place since they are neutral towards his goal of ownership when interacting with the laws contained in $I$. This is done by concurring with the violent events, just as the plaintiff did in clause 16 which corresponds to the defendant's clause 27.

In order to fulfil his goal of ownership the defendant simply has to assume that the testimonies regarding the loan are accurate, as is done in clause 28 . This will make his theory capable of justifying his ownership of the tent since the attempts by the plaintiff to claim ownership using law 2014 BW (clause 9) with its exception, since law $590 \mathrm{BW}$ with its exception (clause 7) is not applicable if the plaintiff was given the tent on loan.

$$
\begin{array}{r}
I_{D 1}=\{\operatorname{Loan}(x, y) \rightarrow \text { TestimonyLoan }(z, x, y) \wedge(x \neq z)\} \\
I_{D 2}=\{\operatorname{Loan}(x, y) \rightarrow \text { TestimonyLoan }(z, x, y) \wedge(x \neq z)\} \\
I_{D 3}=\{\operatorname{Loan}(x, y) \rightarrow \text { TestimonyLoan }(z, x, y) \wedge(x \neq z)\} \\
I_{D 4}=\{\text { Violence }(x, y) \rightarrow \text { TestimonyViolence }(z, x, y) \wedge(y \neq z)\} \\
I_{D 5}=\{\text { Violence }(x, y) \rightarrow \text { TestimonyViolence }(z, x, y) \wedge(y \neq z)\} \\
I_{D 6}=\{\operatorname{Violence}(x, y) \rightarrow \text { TestimonyViolence }(z, x, y) \wedge(y \neq z)\}
\end{array}
$$

Just like the plaintiff, the defendant alternates between two clauses when constructing his input set $I_{D}$, in this case clauses 5 and 3 , both implying that the testimonies of the witnesses are true and are thus indications of a loan and a violent event taking place. He has thus also fulfilled his obligations.

\subsection{Calculation of Coherence}

We will now proceed to calculate the coherence of the "stories" given by the plaintiff and defendant using our measure of coherence introduced in section 3 .

For the six observations $O:\left\{O_{1}, \ldots, O_{6}\right\}$, the plaintiff's theory $T_{P}$ contains five clauses, whereas the the defendant's theory $T_{D}$ contains two clauses. The support sets for the evidence from both sides are as noted in table 3 . We remind the reader that a support set is the subset of a theory that is utilized to account for a given observation. 
Table 3. Support sets for the observations

\begin{tabular}{ccc}
\hline Observation & Plaintiff support set & Defendant support set \\
\hline$O_{1}$ & $\left\{T_{P 5}, T_{P 2}\right\}$ & $\left\{T_{D 2}\right\}$ \\
$O_{2}$ & $\left\{T_{P 5}, T_{P 3}\right\}$ & $\left\{T_{D 2}\right\}$ \\
$O_{3}$ & $\left\{T_{P 5}, T_{P 4}\right\}$ & $\left\{T_{D 2}\right\}$ \\
$O_{4}$ & $\left\{T_{P 5}, T_{P 1}\right\}$ & $\left\{T_{D 1}\right\}$ \\
$O_{5}$ & $\left\{T_{P 5}, T_{P 1}\right\}$ & $\left\{T_{D 1}\right\}$ \\
$O_{6}$ & $\left\{T_{P 5}, T_{P 1}\right\}$ & $\left\{T_{D 1}\right\}$ \\
\hline
\end{tabular}

We remind ourselves that as described in section 4.4 and 4.5, $T_{P}$ and $T_{D}$ are comprised as shown in equation 35 and 36

$$
\begin{gathered}
T_{P}=\left\{T_{P 1}, T_{P 2}, T_{P 3}, T_{P 4}, T_{P 5}\right\} \\
T_{D}=\left\{T_{D 1}, T_{D 2}\right\}
\end{gathered}
$$

The summation of the utility of each of the components of the plaintiff's theory $T_{P}$ over all observations are as shown in equation 37 to 41. These reflect how much each theory component contributed in accounting for all the evidence.

$$
\begin{aligned}
& \sum_{j=1}^{6} U\left(T_{P 1}, T_{P}, I_{P j}, O_{j}\right)=3 \\
& \sum_{j=1}^{6} U\left(T_{P 2}, T_{P}, I_{P j}, O_{j}\right)=1 \\
& \sum_{j=1}^{6} U\left(T_{P 3}, T_{P}, I_{P j}, O_{j}\right)=1 \\
& \sum_{j=1}^{6} U\left(T_{P 4}, T_{P}, I_{P j}, O_{j}\right)=1 \\
& \sum_{j=1}^{6} U\left(T_{P 5}, T_{P}, I_{P j}, O_{j}\right)=6
\end{aligned}
$$

We then average the sum of all the utility of the components over the size of the theory and the number of evidence to derive the coherence measure. The calculation is described in equation 42 ,

$$
C\left(T_{P}, I, O\right)=\frac{1}{6} \times \frac{1}{5} \times(3+1+1+1+6)=0.4
$$

For the case of the defendant, there are only two parts to his story. The summation of the utility of each of the components in the defendant's theory over all observations are as follows. 


$$
\begin{aligned}
& \sum_{j=1}^{6} U\left(T_{D 1}, T_{D}, I_{D j}, O_{j}\right)=3 \\
& \sum_{j=1}^{6} U\left(T_{D 2}, T_{D}, I_{D j}, O_{j}\right)=3
\end{aligned}
$$

The coherence of the defendant's theory can be derived as is done in equation 45 .

$$
C\left(T_{D}, I, O\right)=\frac{1}{6} \times \frac{1}{2} \times(3+3)=0.5
$$

Our coherence measure shows that in this event where the explanations from both sides are considered equally valid, the defendant had provided a more coherent theory to account for the evidence.

The example illustrates a possible application of our notion of coherence in a legal dispute. Both sides were capable of producing a story explaining the evidence, but one did so better than the other as it provided a simpler account for the evidence. This is reflected in the higher coherence value derived from the explanations of the defendant compared to that of the plaintiff. In the following section we will discuss the merits and the shortcomings of this approach in evaluating cases in legal disputes, and identify possible lines of future work.

\section{Discussion and Future Work}

We proposed an approach to evaluate cases in a legal dispute as two rivaling scientific theories. The theories are measured by how well they account for the evidence. The proposed measure of coherence rewards simple theories that account for a large body of evidence, while punishing frivolous theories that regard much of the evidence as exceptions. We gave an example of a known legal dispute from the literature, and showed how the case fits into our framework. In the example, the side that lost due to insufficient evidence also had the less coherent theory.

We note that a key difference between scientific theories and cases in legal disputes is that scientific theories are evaluated primarily on how well they account for the evidence, whereas cases in legal disputes are ultimately concerned with proving a case in order to attain a goal. However, as the case is based on evidence, the quality of the theory in accounting for the evidence is still crucial when proving the case. We argue that our notion of coherence gives rise to an important measure to the quality of a case, and allow rival cases to be compared in a quantitative manner.

Our framework provides only a preliminary and approximate model for evaluating cases with respect to the given evidence. We intentionally chose to simplify the example to illustrate our goal of assessing the coherence of rival theories between the plaintiff and the defendant. In more complicated real-life examples, not all evidence is treated equally, some would be considered worthy of more merit than others, and some would be contradictory. The quality of explanations in 
accounting for a single piece of evidence can also be subjected to debate. The differing merits of evidence and explanations can be modelled by allocating weights to the evidence and also components of the theory in a way similar to the proposal made by Li et al. [7. The merit of cases would then be dependent to the weights associated with each evidence and their explanations. This would be one possible line of future work, but it is beyond the scope of the current paper.

Our measure does not take into account that parts of a chain of conclusion interacts and the final conclusion in such a chain depends on the probability of the chain as a whole. This fact has been noted in Keppens [4. Our measure does, however, capture the effect of corroboratory evidence as described in Walton and Reed [13], since a theory that can account for corroborating accounts of the same observation using only a few clever assumptions in combination with law and common knowledge will be considered "better" according to our measure. Contradictory evidence could be handled by argument-based version of extended logic programming with defeasible priorities such as the one proposed by Prakken and Sartor [9. Incorporating these features into the evaluation of coherence is essential for extending our proposal to more complex legal disputes.

Our evaluation of coherence reflects the importance of the choice of evidence in a legal dispute. As the evidence in a legal dispute is essential for our calculation of coherence of the respective cases, different selection of evidence can change the theories, thus leading to different outcomes in accordance with our coherence measure. Therefore, intelligent allocation of the burden of proof is necessary to collect the relevant evidence in proving the cases, while avoiding material that may not necessarily relate to the case.

Acknowledgments. We would like to thank the referees for their comments, which helped improve this paper considerably. We would also like to thank Mattias Frånberg, Carl Johan Gustavsson, Luke McCrohon and Goran Topić for proof-reading this paper. This work was partially supported by Grant-in-Aid for Specially Promoted Research (MEXT, Japan), and NICTA. NICTA is funded by the Australian Government's Backing Australia's Ability initiative, in part through the Australian Research Council.

\section{References}

1. Amaya, A.: Formal Models of Coherence and Legal Epistemology. Artif. Intell. Law 15, 429-447 (2007)

2. Craig, W.: On Axiomatizability Within a System. The Journal of Symbolic Logic 18, 30-32 (1953)

3. Hage, J.C.: Formalizing Legal Coherence. In: Proc. of 8th International Conference on Artificial Intelligence and Law, pp. 22-31 (2001)

4. Keppens, J.: Towards qualitative approaches to Bayesian evidential reasoning. In: Proceedings of the 11th international conference on Artificial intelligence and law, pp. 17-25 (2007)

5. Kwok, R.B.H., Nayak, A.C., Foo, N.: Coherence Measure Based on Average Use of Formulas. In: Lee, H.-Y. (ed.) PRICAI 1998. LNCS, vol. 1531, pp. 553-564. Springer, Heidelberg (1998) 
6. Kwok, R.B.H., Foo, N., Nayak, A.C.: The Coherence of Theories. In: Proceedings of the 18th Joint International Conference on Artificial Intelligence (IJCAI 2003), Acapulco, Mexico (August 2003)

7. Li, J.J., Kwok, R.B.H., Foo, N.: Coherence of Theories Dependencies and Weights. In: Trends in Logic, vol. 28, pp. 297-318. Springer, Berlin (2009)

8. Prakken, H.: Formalising Ordinary Legal Disputes: A Case Study. Artif. Intell. Law 16, 333-359 (2008)

9. Prakken, H., Sartor, G.: A dialectical model of assessing conflicting arguments in legal reasoning. Artif. Intell. Law 4, 331-368 (1996)

10. Prakken, H., Sartor, G.: Formalising Arguments About the Burden of Persuasion. In: Proc. of ICAIL 2007, pp. 97-106 (2007)

11. Satoh, K., Tojo, S., Suzuki, Y.: Abductive Reasoning for Burden of Proof. In: Proceedings of the 2nd International Workshop on Juris-informatics (JURISIN 2008), Asahikawa, Japan, pp. 93-102 (2008)

12. Walton, D., Macagno, F.: Common Knowledge in Legal Reasoning About Evidence. In: International Commentary on Evidence, vol. 3(1). The Berkeley Electronic Press (2005)

13. Walton, D., Reed, C.: Evaluating Corroborative Evidence. In: Argumentation, vol. 22, pp. 531-553. Springer, Berlin (2008) 